\title{
Inhalation of allergen and diesel exhaust increases urinary eicosanoids associated with impaired lung function and airway hyperresponsiveness: a randomized, double-blinded, human crossover study
}

\author{
Min Hyung Ryu ${ }^{1}$, Cristina Gómez ${ }^{2}$, Agnes Yuen ${ }^{1}$, Craig Wheelock ${ }^{2}$, and Christopher \\ Carlsten $^{1}$ \\ ${ }^{1}$ The University of British Columbia \\ ${ }^{2}$ Karolinska Institutet
}

November 11, 2020

\begin{abstract}
Background. Eicosanoids are potent regulators of homeostasis and inflammation that play an important role in asthma pathophysiology. In a controlled human exposure study, we showed that coexposure to an allergen and diesel exhaust (DE) led to eosinophilic inflammation, impaired airflow, and increased airway responsiveness. Eicosanoids may mediate the mechanism by which these exposures impair lung function. Methods. We conducted a randomized, double-blinded, four-arm crossover study. Fourteen allergen-sensitized participants were exposed to four conditions: filtered air and saline (FA-S; negative control); filtered air and allergen (FA-A; allergen alone); DE and allergen (DE-A; coexposure); and particle-depleted DE and allergen (PDDE-A; coexposure with minimal particles). Quantitative metabolic profiling of urinary eicosanoids was performed using LC-MS/MS. Results. Allergen inhalation increased urinary eicosanoids. The prostacyclin metabolite 2,3-dinor-6-keto-PGF $1 \alpha$ increased with DE-A, but particle depletion (PDDE-A) suppressed this pathway. Baseline airway hyperresponsiveness modified the allergen-induced increase in prostaglandin $\mathrm{D}_{2}$ metabolites (tetranor PGDM and 2,3-dinor-11 $\beta$-PGF $2 \alpha$ ) such that normally responsive individuals showed a greater allergen-induced increase of the two metabolites. Genetic risk scores (GRS) modified the effect of DE-A; those individuals with a high GRS demonstrated a greater increase in isoprostane metabolites following DE-A. Increased urinary leukotriene $\mathrm{E}_{4}$ and tetranor PGDM correlated with increased airway responsiveness, while increased tetranor PGDM also correlated with decline in $\mathrm{FEV}_{1}$. Conclusions. Impaired airflow and increased airway responsiveness upon exposure to DE-A may be attributable to increased levels of leukotrienes and prostaglandins. Variants in genes known to mediate response to pollution appear to modulate these eicosanoid-mediated physiological responses known to contribute to asthma pathophysiology.
\end{abstract}

\section{Hosted file}

20201110 DE3 Eicosanoid Paper_final.pdf available at https://authorea.com/users/374948/ articles/492312-inhalation-of-allergen-and-diesel-exhaust-increases-urinary-eicosanoidsassociated-with-impaired-lung-function-and-airway-hyperresponsiveness-a-randomizeddouble-blinded-human-crossover-study 

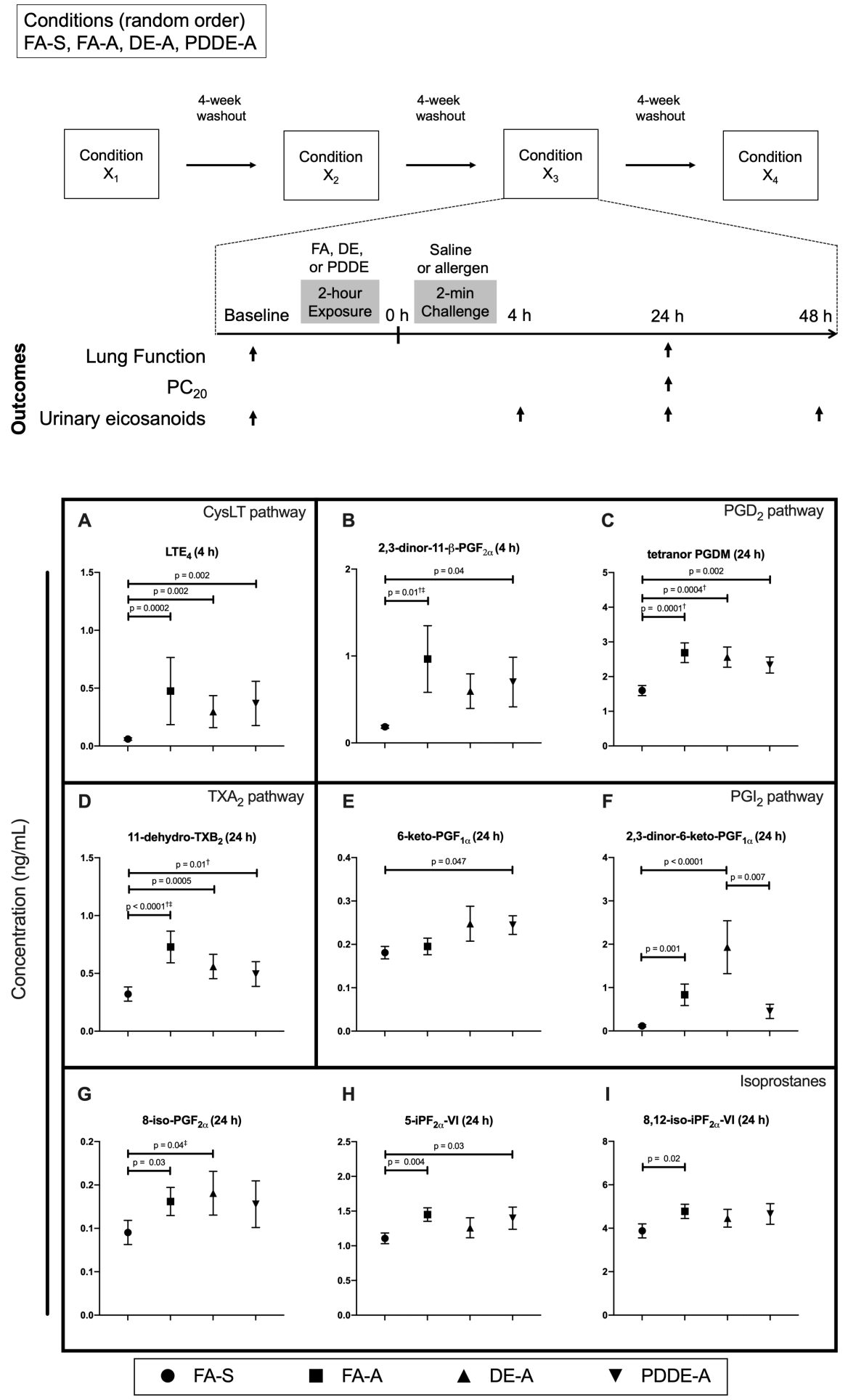